\title{
Main results of the first Data Assimilation Challenge
}

\author{
A. Sciacchitano ${ }^{1 *}$, B. Leclaire ${ }^{2}$, A. Schröder ${ }^{3}$ \\ ${ }^{1}$ Delft University of Technology, Faculty of Aerospace Engineering, Delft, The Netherlands \\ ${ }^{2}$ ONERA, Department of Aerodynamics, Aeroelasticity and Acoustics, Meudon, France \\ ${ }^{3}$ DLR, Institute of Aerodynamics and Flow Technology, Göttingen, Germany \\ *corresponding author: a.sciacchitano@tudelft.nl
}

\begin{abstract}
This work presents the main results of the first Data Assimilation (DA) challenge, conducted within the framework of the European Union's Horizon 2020 project HOMER (Holistic Optical Metrology for Aero-Elastic Research), grant agreement number 769237. The challenge was jointly organised by the research groups of DLR, ONERA and TU Delft. The same synthetic test case as in the Lagrangian Particle Tracking (LPT) challenge (also presented in this symposium) was considered, reproducing the flow in the wake of a cylinder in proximity of a flat wall. The participants were provided with three datasets containing the measured particles locations and their trajectories identification numbers, at increasing tracers concentrations from 0.04 to 1.4 particles $/ \mathrm{mm}^{3}$. The requested outputs were the three components of the velocity, the nine components of the velocity gradient and the static pressure, defined on a Cartesian grid at one specific time instant. The results were analysed in terms of errors of the output quantities and their distributions. Additionally, the performances of the different DA algorithms were compared with that of a standard linear interpolation approach. Although the velocity errors were found to be in the same range as those of the linear interpolation algorithm, typically between $3 \%$ and $12 \%$ of the bulk velocity, the use of the DA algorithms enabled an increase of the measurement spatial resolution by a factor between 3 and 4 . The errors of the velocity gradient were of the order of $10-15 \%$ of the peak vorticity magnitude. Accurate pressure reconstruction was achieved in the flow field, whereas the evaluation of the surface pressure revealed more challenging.
\end{abstract}

\section{Introduction}

In the recent years, three-dimensional velocity measurements by Particle Image Velocimetry (PIV) have evolved from crosscorrelation-based volume analysis (Elsinga et al., 2006; Scarano, 2012) to tracking of individual particles (Particle Tracking Velocimetry, PTV, Malik et al., 1993; Lagrangian Particle Tracking, LPT, Schanz et al., 2016). One of the main advantages of the particle tracking approaches lays in the increased measurement spatial resolution, because a velocity (and acceleration) vector is determined for each and every reconstructed particle, without averaging such information within a spatial subdomain. However, the particle tracking approaches return velocity vectors at the scattered locations where the tracer particles are present. For data reduction purposes, it is often convenient to map such information onto a regular (Cartesian) grid, so as to facilitate the operations required for the computation of relevant flow properties such as the vorticity, the Q- or $\lambda_{2}$-criteria for vortex identification and the shear rate, among others. Additionally, the evaluation of the pressure field via the direct integration of the pressure gradient or the solution of the Poisson equation for pressure (van Oudheusden, 2013) is typically performed on a regular grid, although grid-less approaches for the solution of the Poisson equation have also been proposed (Kunhert and Tiwari, 2001). The conventional techniques to map scattered flow information onto a regular grid involve the use of interpolation (usually linear or cubic interpolation) or spatial averaging of the particles' velocities and accelerations over sub-domains or bins (e.g. adaptive Gaussian windowing technique, AGW, Agüí and Jimenez, 1987). However, these approaches suffer from low spatial resolution, because are incapable to resolve flow wavelengths smaller than the interparticle distance or the bin linear size. As a result, they lead to spatial modulation of the flow field and unresolved or underresolved length-scales, especially for the study of turbulent flows where a wide range of length-scales is present. The use of prior information on the flow physics, e.g. by imposing the conservation of mass for incompressible flows via application of a solenoidal filter to the retrieved velocity field (Schiavazzi et al., 2014; Azijli and Dwight, 2015), has been shown as a viable methodology to attenuate the measurement noise and enhance the accuracy of the measured flow field. More advanced data assimilation approaches have been recently proposed to enforce the compliance of the resulting flow field with the governing equations of fluid motion, aiming at increasing the range of length-scales resolved, possibly beyond the limit of Nyquist criterion. In the FlowFit algorithm introduced by Gesemann et al. (2016), the velocity field is divided into cubic volumes, where it is represented as a weighted sum of $3^{\text {rd }}$ order $3 \mathrm{D}$ base splines. The spline functions are evaluated by solving an optimisation problem, where a cost function is minimised that imposes physical constraints such as the conservation of mass and momentum for incompressible flows. Alternative approaches involve the use of vortex methods (Christiansen, 1973), which make use of the vorticity transport equation at one time instant (Schneiders et al., 2016) or during a short time sequence (Jeon et al., 2018; Scarano et al., 2021; Jeon, 2021) to retrieve a vorticity field compatible with the measured particles' velocities and accelerations. Four-dimensional data assimilation has been proposed recently within the framework of variational methods for computational fluid dynamics (Chandramouli et al., 2020). 
The discussion above highlights the presence of a multitude of approaches aiming at combining flow measurements by LPT and background information on the flow physics to accurately reconstruct the flow field on a regular grid. The aim of the first data assimilation challenge, whose main results are presented in this work, is to comparatively assess the different data assimilation approaches using a database from a simulated experiment, so as to shed light on the capabilities of these approaches and on which parameters and error sources have the largest influence on their performance.

\section{Database description}

The database used for the Data Assimilation challenge is described in detail in the contribution from Leclaire et al. (2021), also presented at the ISPIV 2021. For sake of completeness, a short description of the database is reported hereafter. The database contains the particles' velocities from a synthetic experiment on a wall-bounded wake flow behind a cylinder. The cylinder had a diameter $\mathrm{D}=0.01 \mathrm{~m}$, and was located at $0.01 \mathrm{~m}$ distance from the wall. The fluid used in the simulations was air, but scaling was applied to simulate an experiment in water at a bulk velocity of $V_{\infty}=0.667 \mathrm{~m} / \mathrm{s}$, yielding a turbulent boundary layer of thickness $\delta \approx 60 \mathrm{~mm}$ and a momentum thickness Reynolds number of $\operatorname{Re}_{\theta}=4,500$. The flow domain for the DA challenge was the same as the one used in the LPT challenge (Sciacchitano et al., 2021), and had dimensions of $0.1 m \times 0.05 m \times 0.03 m(\Delta \mathrm{X} \times \Delta \mathrm{Y} \times \Delta \mathrm{Z}$, being $\mathrm{X}, \mathrm{Y}$ and $\mathrm{Z}$ the streamwise, spanwise and wall-normal directions respectively; see Figure 1).
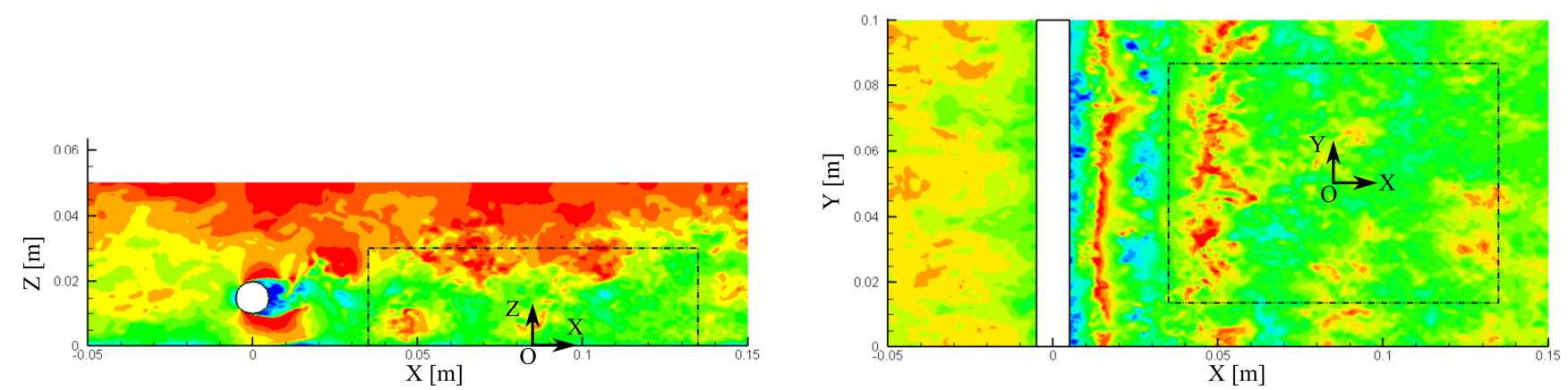

Figure 1: Side view (left) and top view (right) of the flow domain. The dashed rectangle indicates the domain used for the DA challenge. Iso-contours of the instantaneous streamwise velocity component are shown; the flow is in the positive $X$ direction. The origin of the system of axes used for the DA challenge is indicated with $\mathrm{O}$ in the figure.

The particles' positions in 3D space and their trajectories identification numbers were provided to the participants for a sequence of 50 equally-spaced time instants at time separation $\Delta \mathrm{t}=600 \mu \mathrm{m}$. The particles' positions were affected by a 0.1 back-projected pixel $\overline{p x}$ random error (information not disclosed to the participants). The database comprised three different datasets at increasing tracer particles concentrations, as reported in Table 1.

Table 1: Main parameters of the datasets composing the DA database.

\begin{tabular}{cccccc}
\hline $\begin{array}{c}\text { Dataset } \\
\#\end{array}$ & $\begin{array}{c}\text { Equivalent } \\
\text { ppp }^{1}\end{array}$ & $\begin{array}{c}\text { \# of particles in the } \\
\text { fluid domain }\end{array}$ & $\begin{array}{c}\text { Particles } \\
\text { concentration } \\
\left.\text { C [particles } / \mathrm{mm}^{3}\right]\end{array}$ & $\begin{array}{c}\text { Average inter- } \\
\text { particle spacing } \\
\ell[\mathrm{mm}]\end{array}$ & $\begin{array}{c}\text { Normalised inter- } \\
\text { particle spacing } \\
\ell / h\end{array}$ \\
\hline 1 & 0.005 & 6,422 & 0.043 & 1.61 & 4.0 \\
2 & 0.025 & 31,847 & 0.212 & 0.94 & 2.3 \\
3 & 0.160 & 204,280 & 1.362 & 0.52 & 1.3 \\
\hline
\end{tabular}

The participants were requested to provide 13 output quantities on a Cartesian grid of spacing $h=0.4 \mathrm{~mm}$ composed of 251 grid locations along $X$ (from $-50 \mathrm{~mm}$ to $+50 \mathrm{~mm}$ ), 126 along $Y$ (from $-25 \mathrm{~mm}$ to $+25 \mathrm{~mm}$ ) and 76 along $Z$ (from $+0.01 \mathrm{~mm}$ to $+30.01 \mathrm{~mm}$ ), for a total of 2,403,576 grid points. The 13 output quantities were:

- The three components of the velocity vector $\left(V_{X}, V_{Y}, V_{Z}\right)$, in $\mathrm{m} / \mathrm{s}$;

- The nine components of the velocity gradient tensor $\left(\partial V_{X} / \partial X, \partial V_{X} / \partial Y, \partial V_{X} / \partial Z, \partial V_{Y} / \partial X, \partial V_{Y} / \partial Y\right.$, $\left.\partial V_{Y} / \partial Z, \partial V_{Z} / \partial X, \partial V_{Z} / \partial Y, \partial V_{Z} / \partial Z\right)$ in $\mathrm{s}^{-1}$;

- The static pressure $p$ in Pa, relative to the point $(X, Y, Z)=(0,0.2,0.01) \mathrm{mm}$.

All output quantities were requested at the time instant number 25 . The data were analysed in terms of errors of the output quantities (viz. difference from the actual value from the LES simulation at each grid location), their distributions and spectral content.

1 The equivalent ppp is evaluated considering a synthetic experiment as that of the LPT challenge described in Leclaire et al. (2021) and Sciacchitano et al. (2021). 


\section{Participants and approaches}

Four research groups participated to the DA challenge, namely the German Aerospace Centre from Göttingen (DLR), the Kutateladze Institute of Thermophysics in Russia (IOT), Delft University of Technology in the Netherlands (TU Delft, shortly TUD) and the German instrumentation company LaVision GmbH. The approaches employed by these groups are briefly summarised hereafter. As reported in Table 2, differences among the algorithms are already present in the way the particles' locations are fitted to retrieve the positions, velocities and accelerations at time instant $t=25$, which constitute the inputs for the DA approaches.

Table 2: Types and kernel sizes of the fit used to determine the particles' positions, velocities and accelerations.

\begin{tabular}{lcc}
\hline Participant (approach) & Track fit type & Track fit kernel size \\
\hline DLR & Cubic B-spline & $\begin{array}{c}\text { Adaptive, based on the spectral } \\
\text { analysis of the particles' tracks } \\
\text { Adaptive, based on the spectral } \\
\text { analysis of the particles' tracks }\end{array}$ \\
IOT & Cubic B-spline & $7 / 7 / 9$ at ppp $=0.005 / 0.025 / 0.160$ \\
& & $7 / 7 / 9$ at ppp $=0.005 / 0.025 / 0.160$ \\
LaVision (VIC\#-3D) & $2^{\text {nd }}$ order polynomial & 9 \\
LaVision (VIC\#-4D) & $2^{\text {nd }}$ order polynomial & 9 \\
TUD (VIC+) & $2^{\text {nd }}$ order polynomial, 3 iterations & $2^{\text {nd }}$ order polynomial, 3 iterations \\
TUD (TSA) &
\end{tabular}

\subsection{DLR: FlowFit2}

The approach employed by the DLR group is based on the TrackFit and FlowFit2 algorithms, which are described in detail in Gesemann et al. (2016). The main processing steps are the following:

a) Determination of the particles' trajectories according to the provided locations and track-ID data;

b) Spectral analysis of the location-over-time signals to estimate the optimal TrackFit parameters;

c) TrackFit: estimation of the particle trajectories as uniform cubic B-spline curves;

d) Sampling of the particle track B-spline functions at the specified time step (namely 25), including first and second derivatives (velocity and acceleration), as input to FlowFit;

e) FlowFit2: non-linear estimation of velocity and pressure fields as 3D uniform cubic B-splines based on a weighted least-square optimisation that minimises the sum of several squared errors. Those include: the divergence of the velocity field, the gradient of the divergence of the velocity field, deviations between measured and fitted velocities and accelerations, deviations from the pressure Poisson equation, velocity vector Laplacian.

Before the FlowFit2 step, additional virtual particles with zero velocity and acceleration are generated at the wall $(Z=0 \mathrm{~m})$ to comply with the no-slip boundary condition.

\subsection{IOT}

The STB algorithm from the OpenLPT project (Tan et al. 2020) with slight modifications is used by the IOT group. The processing algorithms employed are described in Bobrov et al. (2021) and consists of four main stages:

a) Track approximation by weighted cubic cardinal B-splines (Gesemann et al., 2016), where the weighting coefficients were determined by minimisation of a cost function using the gradient descent method. The velocity and acceleration along the tracks were computed via analytical derivation in time of the B-splines using previously obtained weights;

b) Calculation of the spatial derivatives of the velocity and acceleration via the least-squares method on an unstructured grid, using the approach proposed by Kuhnert and Tiwari (2001);

c) Calculation of the pressure field via iterative joint solution of the Navier-Stokes equations and the Poisson equation for pressure (Kuhnert and Tiwari, 2001);

d) Kriging interpolation of the resulting data onto the output Cartesian grid.

To enhance the accuracy of the estimated pressure field at low seeding concentrations, virtual particles were inserted into the flow domain, using kriging interpolation of the values from the known particles' positions.

\subsection{TUD: VIC+ and TSA}

The TU Delft team made use of two approaches, both based on the Vortex-in-Cell method (Christiansen, 1973). The first approach, named VIC+ (Schneiders and Scarano, 2016), seeks a vorticity field defined at the output Cartesian grid such that a cost function is minimised. The latter depends on the difference between the measured and reconstructed velocities and Lagrangian accelerations at the particles' locations. The velocity field is then obtained from the reconstructed vorticity field via the solution of the Poisson equation. The second approach, named Time-Segment Assimilation (TSA, Scarano et al., 2021) is an evolution of the VIC+ concept which exploits the temporal information from time-resolved measurements. In this case, the vorticity dynamics equation is used to march forward and backward for a finite number of exposures (in total 31 at $\mathrm{ppp}=$ 0.005 and 21 at ppp $=0.025$ ) the first guess of the vorticity field at time $t=0$. The cost function is built as the difference 
between the measured and the reconstructed velocity at the particles' locations along the entire time segment. It should be noted that, due to the high computational cost, the TSA results were only produced for ppp $=0.005$ and 0.025 , and not for $\mathrm{ppp}=0.160$. Also, the pressure field was evaluated only for the VIC+ analysis (and not for the TSA analysis), by solving the Poisson equation for pressure (van Oudheusden, 2013), using Neumann boundary conditions at all boundary points.

\subsection{LaVision: 3D and 4D VIC\#}

Also LaVision GmbH made use of two approaches, one relying only on instantaneous data (3D) and one exploiting the information on the time evolution from time-resolved measurements (4D). The approaches, indicated with VIC\#-3D and VIC\#-4D, respectively, are based on an evolution of the VIC+ algorithm (Schneiders and Scarano, 2016) where additional physical constraints on the divergence of velocity, vorticity, Eulerian acceleration, Lagrangian acceleration and on the momentum equation are imposed (Jeon et al., 2018; Jeon, 2021). A multi-grid approximation was performed so as to decrease the computational cost due to large number of elements in the output Cartesian grid.

\section{Results}

\subsection{Velocity components}

The results of the data assimilation algorithms are expected to exhibit an increasing uncertainty for decreasing seeding concentration, as a consequence of the larger inter-particle distance and therefore lower spatial resolution. For the lowest seeding concentration case ( $\mathrm{ppp}=0.005$ ), Figure 2 compares the streamwise velocity component along several planes in the measurement domain, as well as an iso-surface of the Q-criterion $\left(\mathrm{Q}=80,000 \mathrm{~s}^{-2}\right)$ among ground truth (top-left), participants' results, and linear interpolation of the particles' velocities (bottom right). From the ground truth flow field, the decrease of the velocity towards the wall $(\mathrm{Z}=0)$ due to the presence of the boundary layer is evident. The flow field is clearly turbulent, with small coherent vortical structures visualised via the Q-criterion mainly in the region $\mathrm{X}<0$. The results of the different algorithms are overall rather similar to the ground truth, in that they correctly reproduce the turbulent nature of the boundary layer and close-to-zero velocity at the wall. The result from TUD TSA exhibits more edge effects especially towards the lower limit of $\mathrm{Y}$, where the streamwise velocity component decreases to unphysical values close to zero. The linear interpolation result correctly reproduces the main characteristics of the flow field, although with a larger spatial modulation, thus resulting in smoother velocity contours. However, when the small vortical structures are compared in terms of iso-surfaces of Q-criterion, it is clear that none of the data assimilation algorithms (nor the linear interpolation) is able to correctly capture those due to the limited spatial resolution of the measurement.
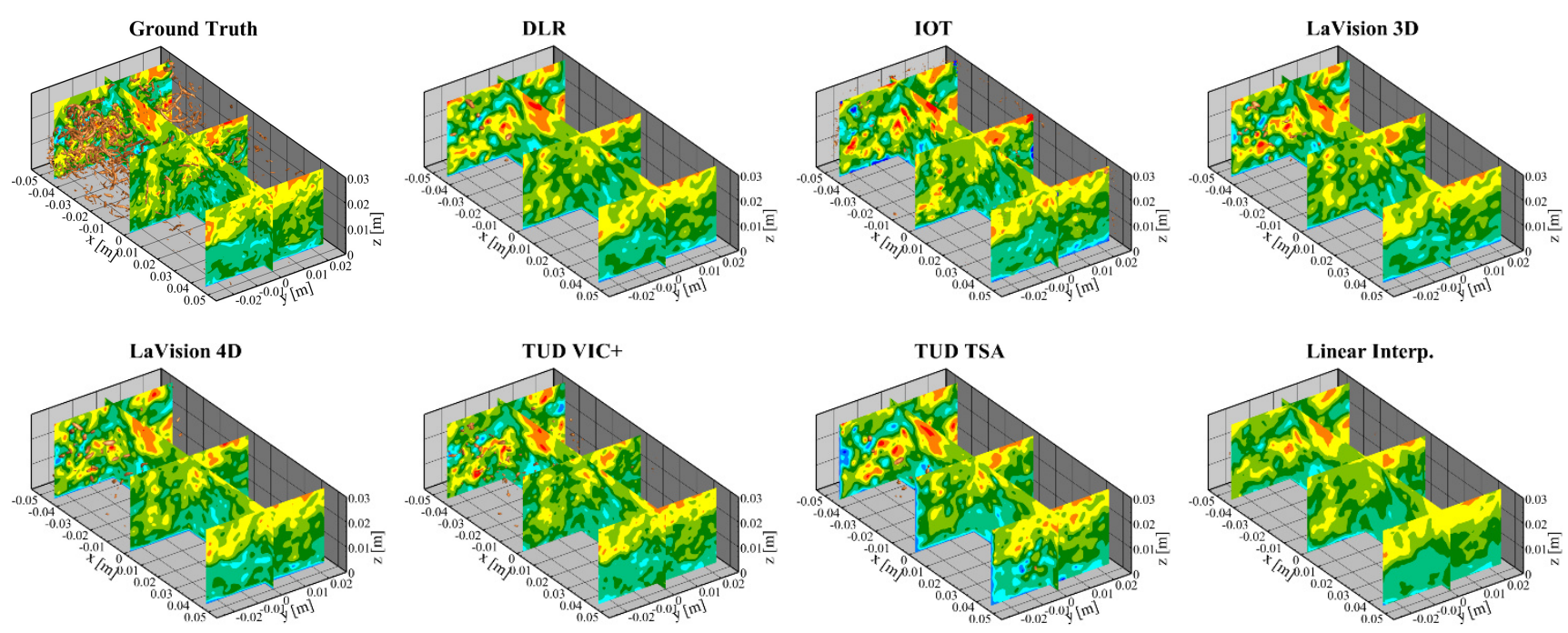

Figure 2: Slices of the streamwise velocity component and of iso-surfaces of $\mathrm{Q}$ criterion $\left(\mathrm{Q}=80,000 \mathrm{~s}^{-2}\right)$ for the ppp $=0.005$ case. The ground truth flow field from the numerical simulations is shown on the top-left. The result from the linear interpolation of the particles velocities onto the Cartesian grid is shown on the bottom-right.

A quantitative analysis of the bias and random errors of the velocity magnitude as a function of the seeding concentration is conducted in the entire measurement domain excluding $4 \mathrm{~mm}$ (ten grid points) from all the outer edges to avoid edge effects. Such analysis is presented in Figure 3. The bias error (Figure 3-left) exhibits little dependence on the ppp, and it typically 
attains values within $0.5 \%$ of the fluid bulk velocity $V_{\infty}$; such errors increase slightly at the lowest ppp, reaching values of $2 \%$ of $V_{\infty}$. The highest errors are encountered with the TUD TSA algorithm, attaining values of up to $4 \%$ of $V_{\infty}$. Also, it is remarked that the linear interpolation algorithm yields similar but slightly larger bias errors to most data assimilation algorithms. The random error, illustrated in (Figure 3-left), shows the expected decrease with increasing seeding concentration. At the lowest ppp, the random error is between 0.06 and $0.085 \mathrm{~m} / \mathrm{s}$ (or $9 \%$ and $13 \%$ of $V_{\infty}$ ), and decreases to about $0.025 \mathrm{~m} / \mathrm{s}$ (roughly $4 \%$ of $V_{\infty}$ ) at the highest ppp. At each seeding concentration, small but systematic differences of about 0.01-0.02 m/s among the different algorithms are recorded. It is noticed that the linear interpolation approach yields random error values of the same order as those of the data assimilation algorithms.
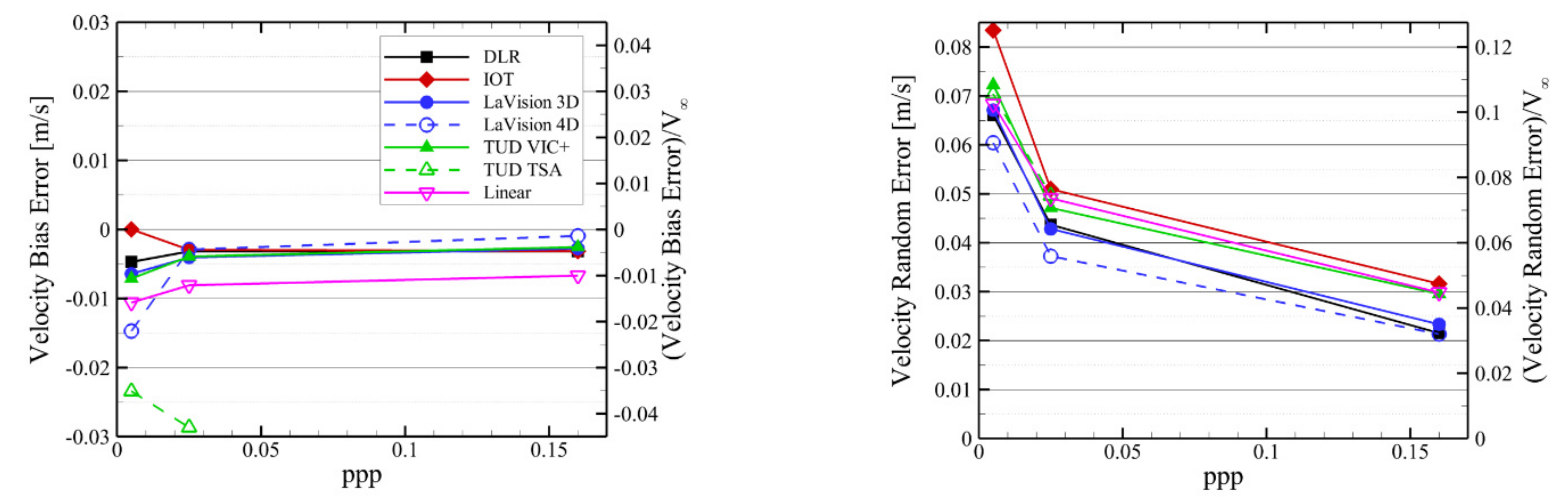

Figure 3: Bias (left) and random (left) error of the velocity magnitude as a function of the ppp. The symbol keys apply to both plots.

To further assess the capabilities of the data assimilation approaches to resolve small scales in the flow field, a spectral analysis is conducted in a region of the flow domain away from the wall, namely for $20 \mathrm{~mm} \leq Z \leq 30 \mathrm{~mm}$. The average power spectral density in such region of the wall-normal velocity component $V_{Z}$ is illustrated in Figure 4 for the three ppp values. As expected, the different algorithms agree well with the ground truth results (thick grey lines) at the lower wavenumbers $k$ (larger wavelengths $\lambda$ ), whereas the agreement worsens for increasing wavenumbers. Also expected is the improved agreement with increasing seeding concentration. At the lowest ppp of 0.005 (Figure 4-left), the linear interpolation result departs from the ground truth already at a wavenumber of $50 \mathrm{~m}^{-1}$ (or wavelength of $20 \mathrm{~mm}$, that is more than 12 times larger than the average inter-particle distance). In contrast, the data assimilation algorithms follow the ground truth result up to $k \approx 200 \mathrm{~m}^{-1}(\lambda \approx 5 \mathrm{~mm})$, thus yielding an increase of the range of resolved length scales by factor 4 with respect to the linear interpolation. Similar trends are retrieved also at the higher seeding concentrations (Figure 4-middle and -right), with improved agreement with the ground truth result. In particular, at the highest ppp of 0.16 , most data assimilation algorithms capture correctly the power spectrum up to $k \approx 300 \mathrm{~m}^{-1}(\lambda \approx 3 \mathrm{~mm})$, whereas the linear interpolation result starts departing from the ground truth already around $k \approx 100 \mathrm{~m}^{-1}(\lambda \approx 10 \mathrm{~mm})$. Hence, based on this spectral analysis, it can be concluded that the data assimilation algorithms increase the range of resolvable length scales by factor 3 to 4 with respect to the standard interpolation, and that the smallest fluctuations correctly captured occur at a length scale that is 3 to 6 time larger than the average inter-particle distance.
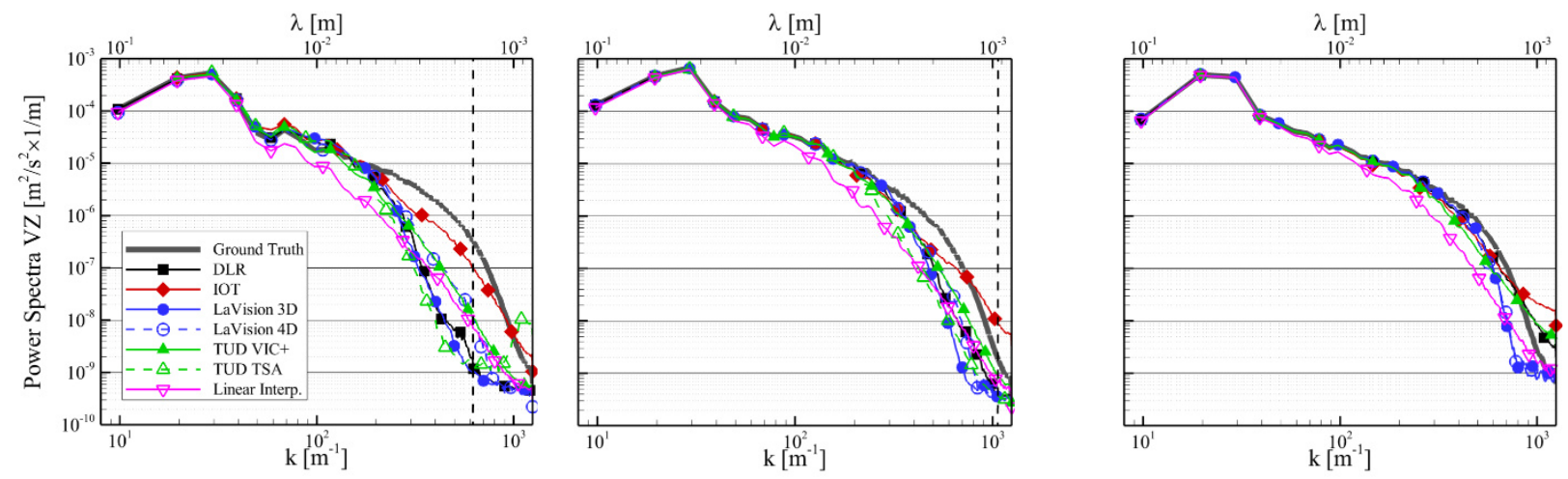

Figure 4: Power spectral density of the wall-normal velocity component $\mathrm{V}_{\mathrm{Z}}$, averaged in the region $20 \mathrm{~mm} \leq \mathrm{Z} \leq 30 \mathrm{~mm}$. Left: $\mathrm{ppp}=0.005$; middle: $\mathrm{ppp}=0.025$; right: $\mathrm{ppp}=0.16$. The bottom horizontal axis represents the wavenumber, while the top horizontal axis represents the corresponding wavelength. The dashed vertical line, when present, corresponds to the wavenumber (or wavelength) associated with the average inter-particle spacing. The symbol keys apply to all plots. 


\subsection{Velocity gradient components}

The evaluation of the components of the velocity gradient is notoriously more challenging than that of the velocity itself for two main reasons: first, under-resolved or unresolved length scales result in the underestimation of the spatial derivatives of the velocity; second, the spatial derivative operator acts as a high-pass filter onto the velocity field, thus yielding a decrease of the measurement signal-to-noise ratio in presence of uncorrelated noise. The accuracy of the evaluation of the velocity gradient components is assessed in Figure 5 via the analysis of the vorticity magnitude for the case ppp $=0.005$. The ground truth vorticity field presents vorticity peaks up to $1000 \mathrm{~Hz}$ attributed to small-scale vortical structures especially in the region closer to the cylinder $(\mathrm{X}<0)$. All considered algorithms exhibit a significant modulation of the vorticity field, yielding vorticity peak values seldom exceeding $500 \mathrm{~Hz}$. The highest modulation occurs with the IOT algorithm and, as expected, when using the linear interpolation approach.
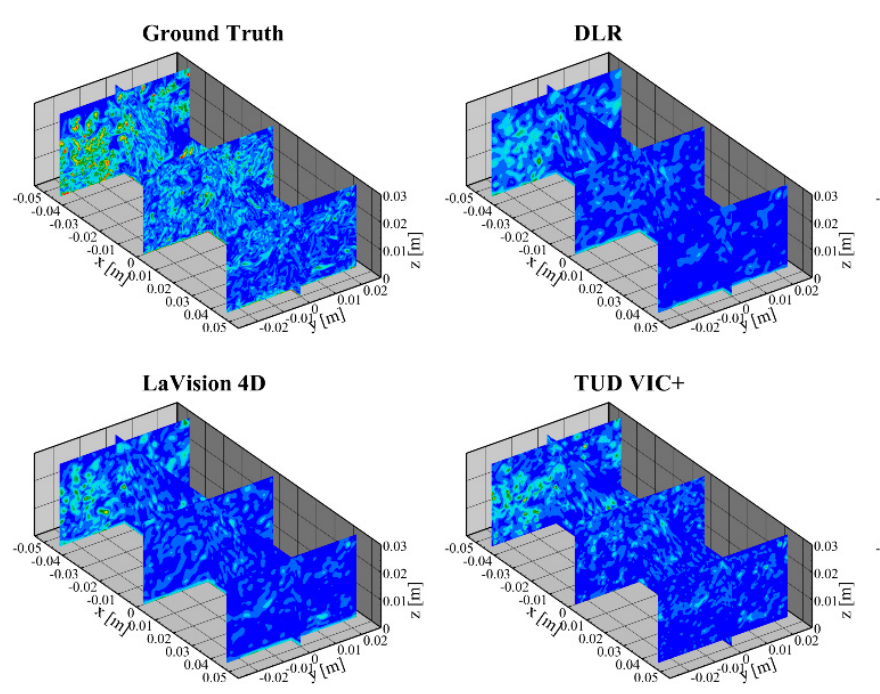

$|\omega|[\mathrm{Hz}]: \quad 1002003004005006007008009001000$
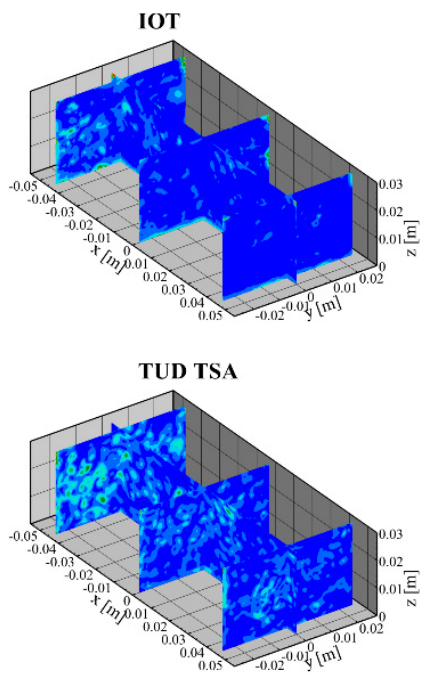
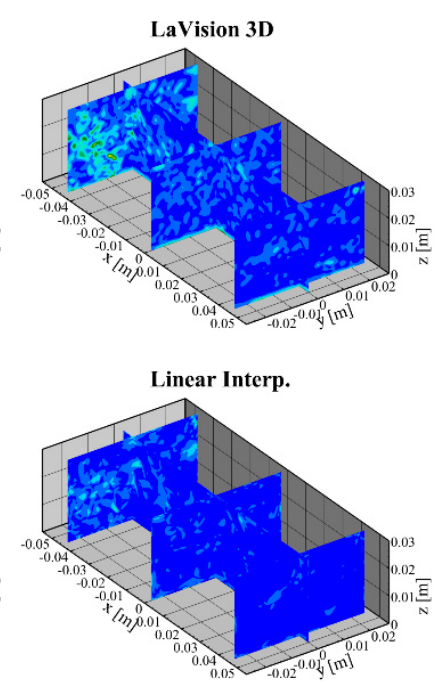

Figure 5: Slices of the vorticity magnitude for the $\mathrm{ppp}=0.005$ case. The ground truth flow field from the numerical simulations is shown on the top-left. The vorticity result from the linear interpolation of the particles velocities onto the Cartesian grid is shown on the bottomright.
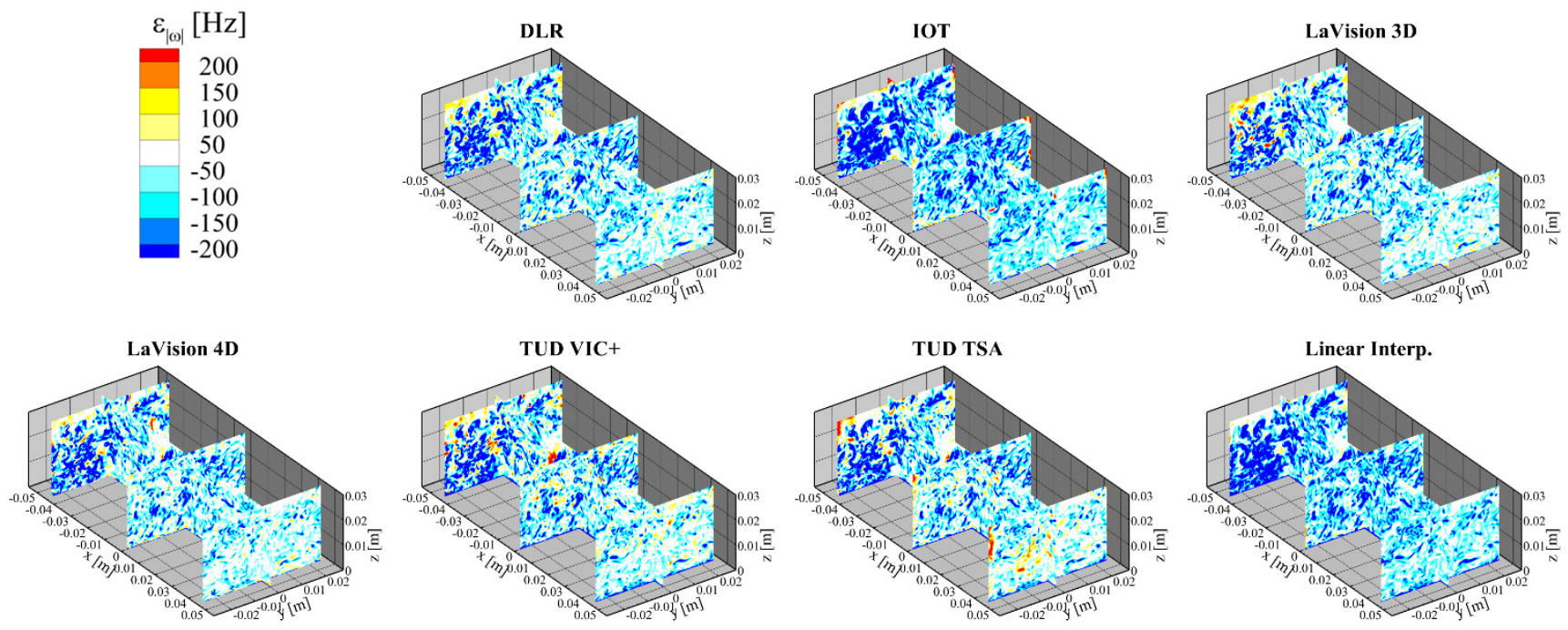

Figure 6: Error of the vorticity magnitude obtained with the different algorithms, for the case ppp $=0.005$.

The contours of the vorticity error magnitude, presented in Figure 6, confirm that the vorticity errors are of the order of $100 \mathrm{~Hz}$ or $10 \%$ of the peak vorticity. These errors are mainly negative because the algorithms tend to underestimate the actual vorticity. The maximum errors are encountered closer to the cylinder, that is at lower values of the streamwise coordinate $\mathrm{X}$, where small vortical structures of high swirling strength are present. From the visual analysis of the contours of Figure 6 , 
small differences are noticed among the different algorithms, with lower vorticity errors for DLR and LaVision 4D algorithms and larger errors for the IOT and the linear interpolation approaches.

Similar to the velocity, a quantitative analysis of the vorticity magnitude error is conducted in terms of mean bias and random components in the entire measurement domain, excluding a region of $4 \mathrm{~mm}$ thickness at the outer edges. The results of this analysis are shown in Figure 7. As expected, there is a clear trend of increasing performance when increasing the seeding concentration. As discussed above, the mean bias errors (Figure 7-left) are negative as a consequence of the spatial modulation of the velocity field that yields underestimated vorticity values. The DLR algorithm exhibits the best performance in terms of mean bias errors, with error values between $-80 \mathrm{~Hz}$ at the lowest ppp and $-20 \mathrm{~Hz}$ at the highest ppp. Similar values are retrieved also with the LaVision algorithms and the TU Delft VIC+ approach. In contrast, the IOT algorithm and the linear interpolation approach return bias error values between -120 and $-80 \mathrm{~Hz}$. The random error component, illustrated in Figure 7-right, exhibits values of around $150 \mathrm{~Hz}$ at $\mathrm{ppp}=0.005$, with small differences among the different algorithms. At higher seeding concentrations, a significant error reduction is retrieved down to $80 \mathrm{~Hz}$ with the DLR and LaVision algorithms; in contrast, for the other algorithms the random errors remain above $110 \mathrm{~Hz}$ (IOT algorithm) or even close to $130 \mathrm{~Hz}$ (TU Delft VIC+ and linear interpolation approaches). Considering that the ground truth peak vorticity attains values of about $1000 \mathrm{~Hz}$, it can be concluded that, using the best data assimilation algorithms at the highest ppp level of 0.160 , the vorticity error values are of the order of $2 \%$ and $8 \%$ for the bias and random components, respectively. However, these errors increase to $8 \%$ and $13 \%$, respectively, when less performing data assimilation algorithms are employed.
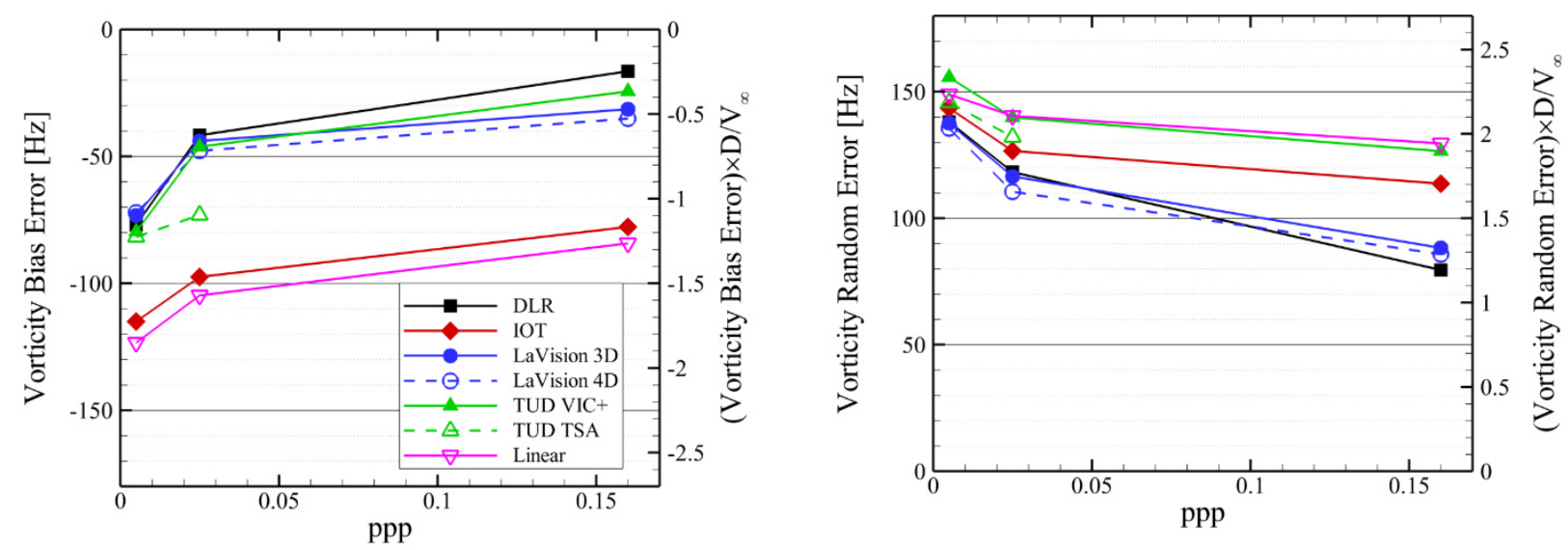

Figure 7: Bias (left) and random (left) error of the vorticity magnitude as a function of the ppp. The symbol keys apply to both plots.

For sake of completeness, the histograms of the velocity divergence are computed, so as to evaluate the accuracy also of the diagonal terms of the velocity gradient tensor. Being the flow incompressible, the velocity divergence is expected to be null, and the histograms are theoretically Dirac pulses centered at zero. The results, illustrated in Figure 8, show that the LaVision 3D and 4D algorithms achieve close-to-zero divergence at all ppp values. Larger values of the velocity divergence are estimated with the DLR algorithm and the TU Delft approaches, typically within 1 or $2 \mathrm{~Hz}$. In contrast, the IOT and the linear interpolation approaches return a much broader distribution of the velocity divergence, which implies a lower degree of agreement of the measured flow field with the continuity equation.
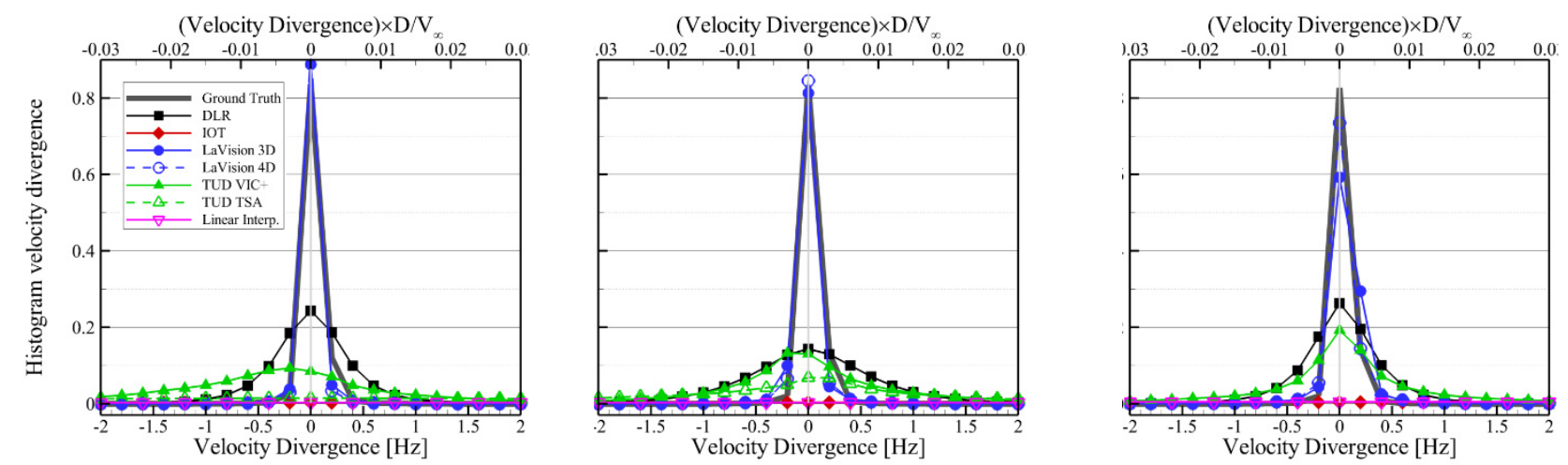

Figure 8: Histograms of the velocity divergence for $\mathrm{ppp}=0.005$ (left), 0.025 (middle) and 0.16 (right). The symbol keys apply to all plots. 


\subsection{Static pressure}

The pressure gradient is related to the Lagrangian acceleration via the Navier-Stokes equations, and then integrated either directly or solving the Poisson equation for pressure (van Oudheusden, 2013). Hence, errors in the Lagrangian acceleration propagate to the pressure, although the integration operator is expected to attenuate the contribution of the random errors. Figure 9 illustrates the static pressure field in a plane close to the centerline ( $Y=-0.2 \mathrm{~mm})$ for the lowest seeding density case (ppp $=0.005$ ), along with the corresponding error fields. The ground truth field shows the presence of two large lowpressure blobs, at $X=-0.03 \mathrm{~m}$ and $\mathrm{X}=0.015 \mathrm{~m}$ respectively, associated with vortices shed by the cylinder. The minimum pressure within these regions is of about $-110 \mathrm{~Pa}$ and $-70 \mathrm{~Pa}$, respectively, corresponding to $50 \%$ and $30 \%$ of the free-stream dynamic pressure $\left(q_{\text {inf }}=221.8 \mathrm{~Pa}\right.$ ). Additionally, small flow features with low pressure are present near the upstream edge of the measurement domain. All algorithms are capable to reproduce the largest low-pressure blob at $\mathrm{X}=-0.03 \mathrm{~m}$, although not always with the correct pressure magnitude. In particular, the IOT algorithm yields a minimum pressure attenuated by $50 \%$, whereas the linear interpolation result ${ }^{2}$ overestimates the pressure peak by $100 \%$. The other algorithms return correct values of the pressure peak typically within $10 \%$. The second pressure blob, located at $X=0.015 \mathrm{~m}$, is not captured by the IOT algorithm, whereas all other algorithms correctly reproduce it. The error fields, presented in the second column of Figure 9, show that the errors are mainly random, with peaks as high as $50 \mathrm{~Pa}$ or over $20 \%$ of the free-stream dynamic pressure. The IOT algorithm and the linear interpolation approach exhibit larger bias errors in correspondence of the pressure peak at $\mathrm{X}=-0.03 \mathrm{~m}$, which is respectively underestimated and overestimated by the two approaches.

The quantitative analysis of the errors, illustrated in Figure 10, confirms that the random errors dominate over the bias errors. The latter are typically in the range [- 5, 5] $\mathrm{Pa}$, except for the IOT algorithm, exceeding the values of $\pm 5 \mathrm{~Pa}$ for $\mathrm{ppp}=0.025$, and the linear interpolation approach, exceeding the values of $\pm 5 \mathrm{~Pa}$ for all the concentrations. As expected, the accuracy of the pressure reconstruction increases with the seeding density, thus yielding a reduction of the random error component (Figure 10-middle). However, the random errors curves decrease rapidly up to $\mathrm{ppp}=0.025$, whereas they flatten for higher seeding concentrations. At ppp $=0.16$, the random errors from the different algorithms range between $4 \mathrm{~Pa}$ (less than $2 \%$ of $q_{\text {inf, }}$ achieved with the DLR algorithm) and $18 \mathrm{~Pa}$ (8\% of $q_{\mathrm{inf}}$, obtained with the linear interpolation approach). Also, it is noticed that the use of temporal information in the data assimilation algorithm slightly improves the pressure reconstruction (see comparison between LaVision 3D and LaVision 4D results, where the latter always yields lower random errors).The cross-correlation coefficients between the participants' results and the ground-truth pressure field (Figure 10-right) confirm the capability of the data assimilation algorithm to accurately reconstruct the larger-scale features in the pressure field. For the two higher seeding concentrations, the cross-correlation coefficient is close to or above 0.8 , with values even exceeding 0.95 especially at $\mathrm{ppp}=0.160$. As anticipated, the pressure reconstruction is more challenging at the lowest seeding concentration due to the low measurement spatial resolution; in these conditions, the cross-correlation coefficients range between 0.5 (IOT algorithm) and 0.93 (LaVision 4D algorithm).
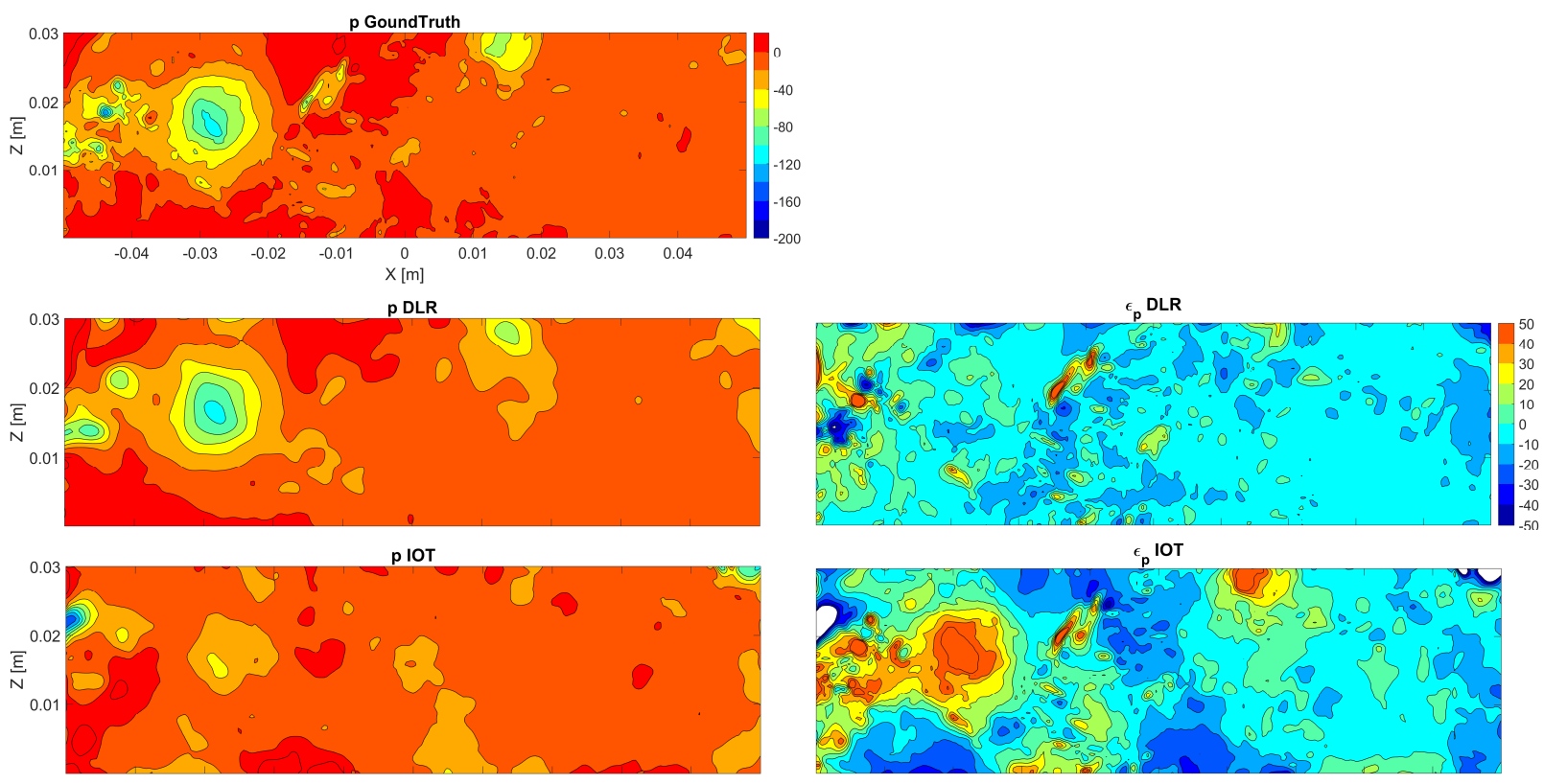

$\epsilon_{\mathrm{p}}$ IOT

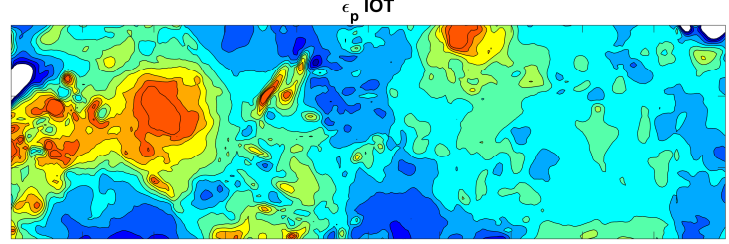

2 The linear interpolation result is obtained by first computing the velocity and Lagrangian acceleration fields based on linear interpolation of the particles' information, then computing the pressure gradient as $\nabla p=-\rho D V / D t$, and finally solving the Poisson equation for pressure. 

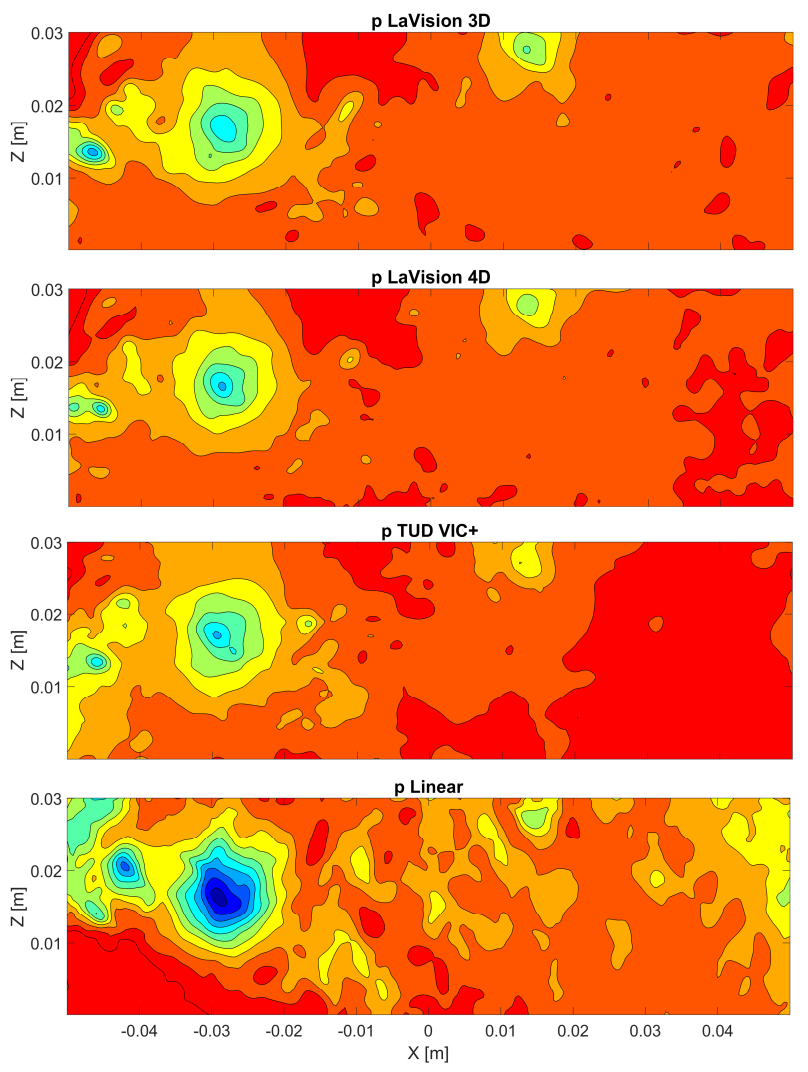

Figure 9: Static pressure field (left column) and error of the static pressure (right column) at the plane $\mathrm{Y}=-0.2 \mathrm{~mm}$, for the case ppp $=0.005$. First row: ground truth result. The values in the colorbars are in Pascal.
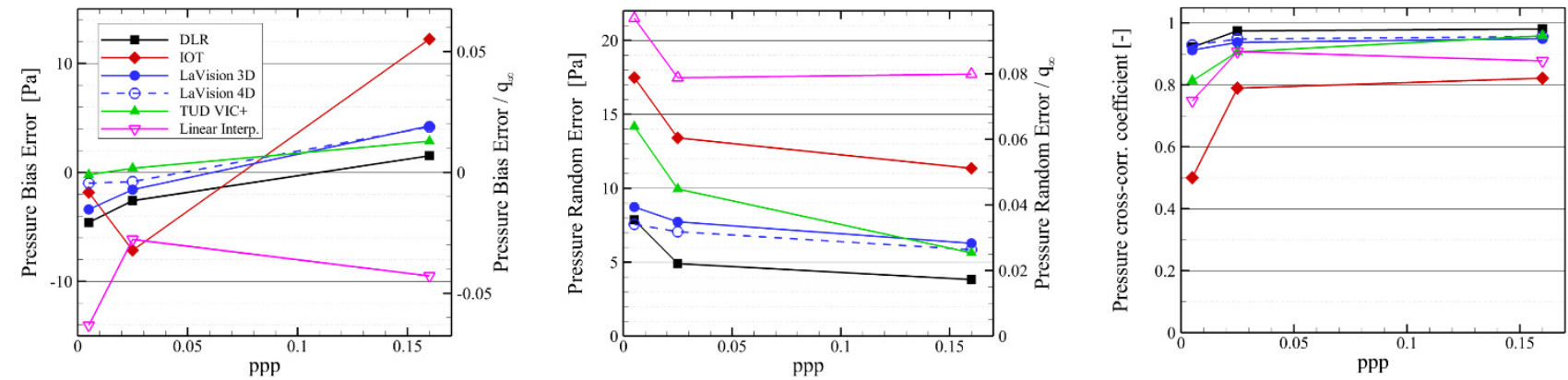

Figure 10: Mean bias error (left), random error (middle) and cross-correlation coefficient with respect to the ground truth (right) of the static pressure, evaluated at plane $\mathrm{Y}=-0.2 \mathrm{~mm}$, as a function of the ppp. The errors and cross-correlation coefficient are evaluated over the entire measurement domain, excluding a border of $4 \mathrm{~mm}$ (10 grid points) at the outer edges. The symbol keys apply to all plots.

The evaluation of the static pressure on the surface of solid objects is of great relevance in aerodynamics and fluid-structure interaction problems, because it enables to characterize the spatial distribution of the aerodynamic loads. Unfortunately, computing the surface pressure often involves even more challenges than evaluating the static pressure in the flow field, because of the small magnitude of the wall-pressure fluctuations, the large velocity gradients in the boundary layer, and the presence of unwanted light reflections. The ground-truth surface pressure field, illustrated in Figure 11-top for the case $\mathrm{ppp}=0.005$, confirms that indeed the pressure variations on the surface are a small fraction of those in the flow field; smallscale flow structures are visible, with pressure values varying between -30 and $30 \mathrm{~Pa}$, with the pressure generally decreasing along the streamwise direction. The results of the different participants, shown in the first column of Figure 11, confirm the complexity of the surface pressure reconstruction problem. At this low seeding concentration value, none of the algorithms is able to correctly reconstruct the small-scale pressure fluctuations encountered in the ground-truth flow field. The DLR result is the closest to the actual pressure field and reproduces the increase of pressure along the streamwise direction, although with strong modulation of the small flow structures. The results from the LaVision 3D and LaVision 4D algorithms strongly attenuate the pressure fluctuations to the range between -5 and $5 \mathrm{~Pa}$. The other approaches, instead, yield a very noisy surface pressure field, with limited agreement with the actual pressure field. For those algorithms, the errors on the estimated pressure, illustrated in the second column of Figure 11, even exceed the actual pressure fluctuations. 

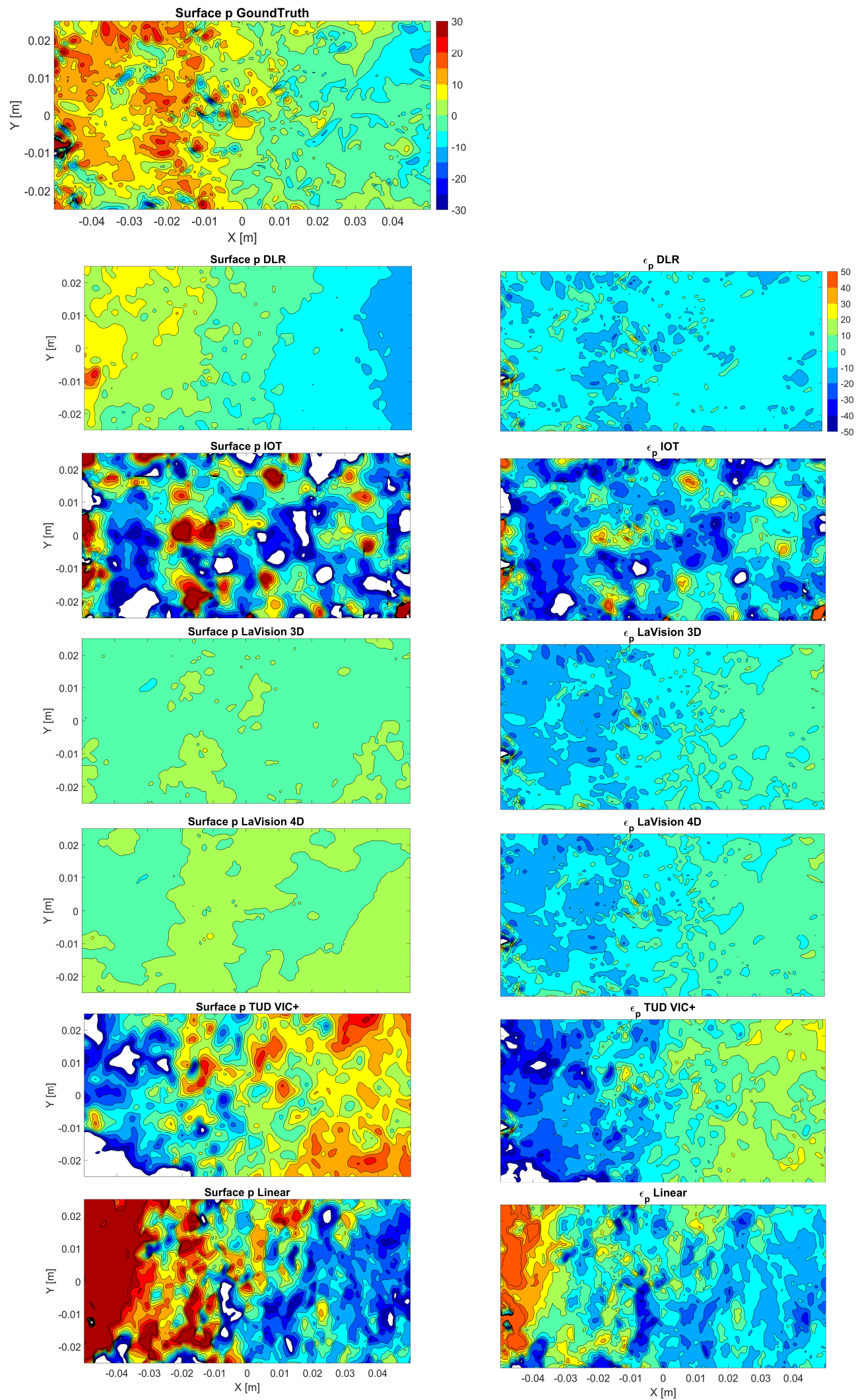

Figure 11: Surface static pressure field (left column) and error of the static pressure (right column), evaluated at $\mathrm{Z}=0.01 \mathrm{~mm}$ from the wall, for the case ppp $=0.005$. First row: ground truth result. The values in the colorbars are in Pascal. 
The quantitative analysis of the mean bias error, random error and cross-correlation coefficient with the ground-truth surface pressure field is presented in Figure 12. The surface pressure errors are in a similar range as the errors in the rest of the flow field shown in Figure 10 (bias errors: between -10 and $10 \mathrm{~Pa}$; random errors: between 5 and $20 \mathrm{~Pa}$ ). However, as a consequence of the smaller magnitude of the surface pressure fluctuations, the cross-correlation coefficient drops significantly to values below 0.8 . The largest cross-correlation coefficient $(0.8)$ is obtained with the DLR algorithm, and is rather independent of the seeding density; in contrast, the other approaches return cross-correlation coefficient values below 0.6, which further drop at the lowest seeding concentration, confirming the poor surface pressure reconstruction accuracy in this condition.

Finally, the results of the spectral analysis on the surface pressure are illustrated in Figure 13 for the three ppp levels. A clear trend is visible of increasing agreement between ground-truth and participants' results at increasing seeding concentration. However, some algorithms (DLR, LaVision 3D and LaVision 4D) underestimate the pressure fluctuations at all wave numbers, whereas others (IOT and linear interpolation) tend to overestimate them, thus resulting in more noisy pressure fields. The pressure spectrum of the TUD VIC+ algorithm agrees well with the ground-truth result at the two larger seeding concentrations, whereas at $\mathrm{ppp}=0.005$ it overestimates the pressure fluctuations at the lower wave numbers $\left(k<20 \mathrm{~m}^{-1}\right)$, and underestimates them at the higher wave numbers.
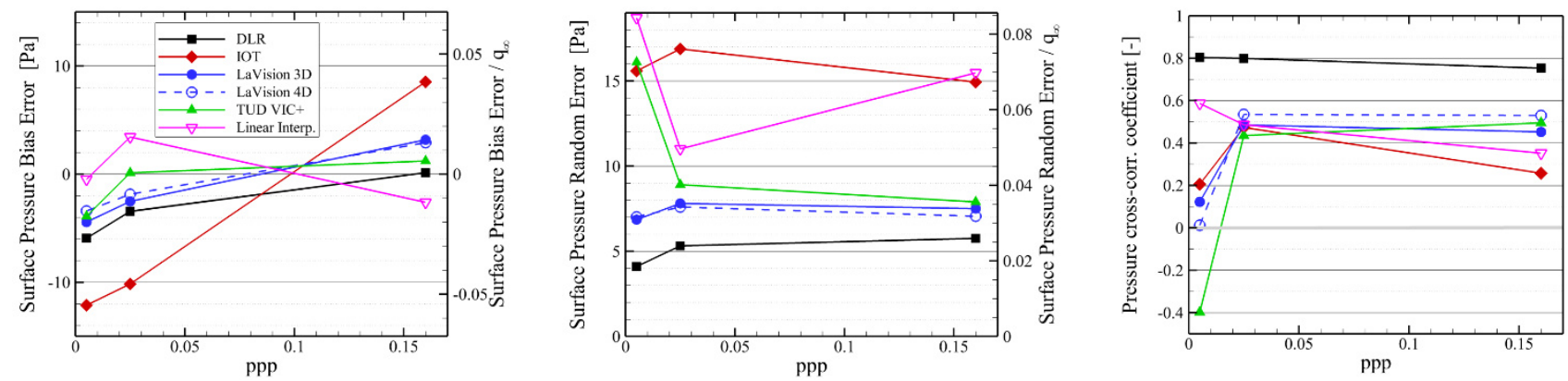

Figure 12: Mean bias error (left), random error (middle) and cross-correlation coefficient with respect to the ground truth of the surface pressure, evaluated in the plane $\mathrm{Z}=0.01 \mathrm{~mm}$, as a function of the ppp. The symbol keys apply to all plots.
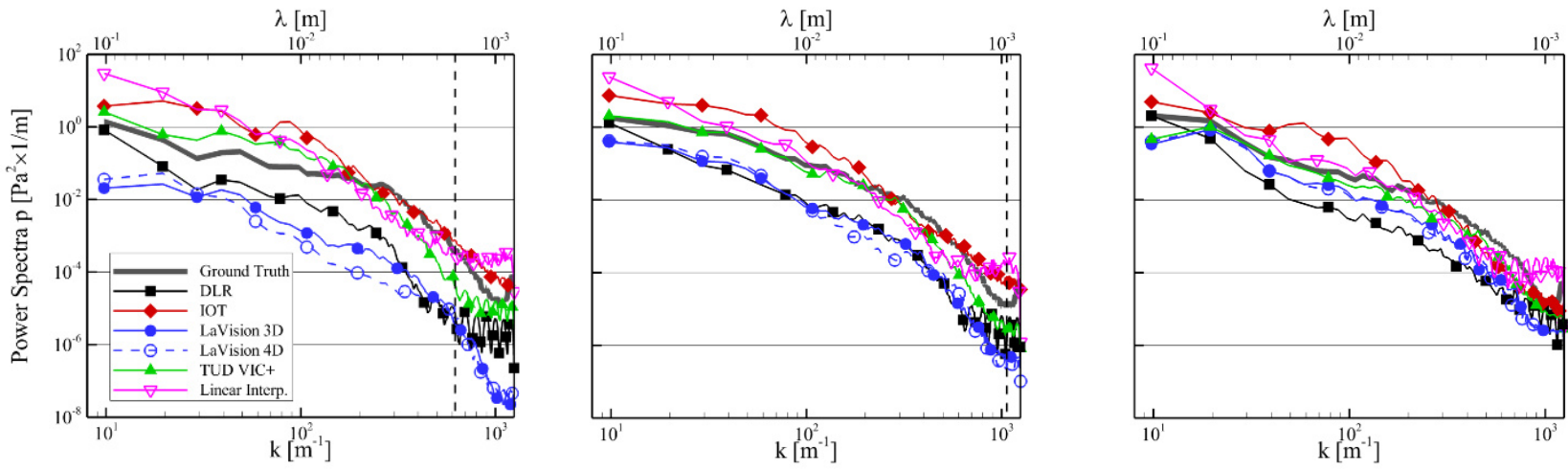

Figure 13: Power spectral density of the surface static pressure, evaluated in the plane $Z=0.01 \mathrm{~mm}$. Left: $\mathrm{ppp}=0.005$; middle: $\mathrm{ppp}=0.025$; right: $\mathrm{ppp}=0.16$. The bottom horizontal axis represents the wavenumber, while the top horizontal axis represents the corresponding wavelength. The dashed vertical line, when present, corresponds to the wavenumber (or wavelength) associated with the average interparticle spacing. The symbol keys apply to all plots.

\section{Conclusions}

This work presents the main results of the Data Assimilation challenge organised within the framework of the European Union's Horizon 2020 project HOMER (Holistic Optical Metrology for Aero-Elastic Research). The challenge made use of a synthetic experiment, presented in another contribution to this symposium (Leclaire et al., 2021), reproducing the wallbounded flow in the wake of a cylinder. The particles' positions along their trajectories were provided to the participants, in three datasets reproducing the seeding concentration levels corresponding to ppp $=0.005,0.025$ and 0.16 . The output quantities were the three velocity components, the nine components of the velocity gradient tensor and the static pressure, all defined in a Cartesian grid of $h=0.4 \mathrm{~mm}$ grid spacing.

Four research groups took part to the DA challenge, namely DLR, IOT, LaVision GmbH and TU Delft. The latter two groups submitted results with two algorithms each. For what concerns the estimated velocity, errors between $3 \%$ and $12 \%$ of the bulk velocity $V_{\infty}$ were obtained, depending on the seeding concentration and the data assimilation algorithm. These errors are of similar magnitude as those achieved when using the conventional linear interpolation of the particles' velocities onto the 
$14^{\text {th }}$ International Symposium on Particle Image Velocimetry - ISPIV2021

August 1-4, 2021

output Cartesian grid. However, the spectral analysis revealed that the use of the data assimilation algorithms enables to increase the range of resolved length scales by factors 3 to 4 with respect to the linear interpolation approach. The analysis of the velocity gradients highlighted the presence of bias and random errors of $100-150 \mathrm{~Hz}$, or 10-15\% of the typical vorticity magnitude peaks. As expected, both error components decrease with increasing seeding concentration; however, even at the highest ppp of 0.16 , bias and random errors exceeding $20 \mathrm{~Hz}$ and $80 \mathrm{~Hz}$, respectively, are obtained. Finally, the evaluated pressure featured bias errors within $\pm 10 \mathrm{~Pa}$ and random errors between $5 \mathrm{~Pa}$ and $20 \mathrm{~Pa}$. A better agreement with the actual pressure field (cross-correlation coefficient exceeding 0.95) was achieved away from the wall, whereas on the solid surface the agreement decreased (cross-correlation coefficient below 0.8) due to the lower magnitude of the pressure fluctuations.

\section{Acknowledgments}

This work has been carried out in the context of the HOMER (Holistic Optical Metrology for Aero-Elastic Research) project, funded by the European Union's Horizon 2020 research and innovation programme under grant agreement No 769237. The data processing performed by the research groups who participated in the challenge is kindly acknowledged.

\section{References}

Agüi J and Jimenez J (1987) On the performance of Particle Tracking Velocimetry. Journal of Fluid Mechanics 185:447-468

Azijli I and Dwight RP (2015) Solenoidal filtering of volumetric velocity measurements using Gaussian process regression. Experiments in Fluids 56(11), 1-18

Bobrov M, Hrebtov M, Ivashchenko V, Mullyadzhanov R, Seredkin A, Tokarev M, Zaripov D, Dulin V and Markovich D (2021) Pressure evaluation from Lagrangian particle tracking data using a grid-free least-squares method. Measurement Science and Technology 32, 084014

Chandramouli P, Mémin E and Heitz D (2020). 4D large scale variational data assimilation of a turbulent flow with a dynamics error model. Journal of Computational Physics 412, 109446

Christiansen IP (1973) Numerical simulation of hydrodynamics by the method of point vortices. Journal of Computational Physics 13(3), 363-379

Elsinga GE, Scarano F, Wieneke B and van Oudheusden BW (2006) Tomographic particle image velocimetry. Experiments in fluids 41(6), 933-947.

Gesemann S, Huhn F, Schanz D and Schröder A (2016) From noisy particle tracks to velocity, acceleration and pressure fields using B-splines and penalties. In 18th international symposium on applications of laser and imaging techniques to fluid mechanics, Lisbon, Portugal (pp. 4-7)

Jeon YJ, Schneiders JFG, Müller M, Michaelis D and Wieneke B (2018) 4D flow field reconstruction from particle tracks by VIC+ with additional constraints and multigrid approximation. In Proceedings 18th International Symposium on Flow Visualization, ETH Zurich

Jeon YJ (2021) Eulerian time-marching in Vortex-In-Cell (VIC) method: reconstruction of multiple time-steps from a single vorticity volume and time-resolved boundary condition. In 14th International Symposium on Particle Image Velocimetry - ISPIV 2021, August 1-5, 2021

Kuhnert J and Tiwari S (2001) Grid free method for solving the Poisson equation. Fraunhofer-Institut für Techno- und Wirtschaftsmathematik, https://nbn-resolving.org/urn:nbn:de:hbz:386-kluedo-12885

Leclaire B, Mary U, Liazun C et al. (2021) First Lagrangian Particle Tracking and Data Assimilation challenge: datasets description and evolution to an open online benchmark. In 14th International Symposium on Particle Image Velocimetry - ISPIV 2021, August 1-5, 2021

Malik NA, Dracos T and Papantoniou DA (1993) Particle tracking velocimetry in three-dimensional flows. Experiments in Fluids 15(4), 279-294

Scarano F (2012) Tomographic PIV: principles and practice. Measurement Science and Technology 24(1), 012001

Scarano F, Schneiders JFG, Gonzalez Saiz G and Sciacchitano A (2021) Dense velocity reconstruction with VIC-based timesegment assimilation, under review in Experiments in Fluids

Schanz D, Gesemann S and Schröder A (2016) Shake-The-Box: Lagrangian particle tracking at high particle image densities. Experiments in Fluids 57(5), 1-27

Schiavazzi D, Coletti F, Iaccarino G and Eaton JK (2014) A matching pursuit approach to solenoidal filtering of threedimensional velocity measurements. Journal of Computational Physics 263, 206-221

Schneiders JFG and Scarano F (2016) Dense velocity reconstruction from tomographic PTV with material derivatives. Experiments in Fluids 57(9) 1-22 
$14^{\text {th }}$ International Symposium on Particle Image Velocimetry - ISPIV2021

August 1-4, 2021

Sciacchitano A, Leclaire B and Schröder A (2021) Main results of the first Lagrangian Particle Tracking Challenge. In 14th International Symposium on Particle Image Velocimetry - ISPIV 2021, August 1-5, 2021

Tan S, Salibindla A, Masuk A U M and Ni R (2020) Introducing OpenLPT: new method of removing ghost particles and high-concentration particle shadow tracking. Experiments in Fluids 61: 47

van Oudheusden BW (2013) PIV-based pressure measurement. Measurement Science and Technology 24(3), 032001 